\title{
TURISMO, COCINAS, SABORES Y SABERES LOCALES Y REGIONALES SOSTENIBLES EN PERÚ
}

\author{
SUSTAINABLE TOURISM, LOCAL AND REGIONAL KITCHENS, \\ TASTES, AND SABERES IN PERU
}

Tirso Gonzales

Pontificia Universidad Católica del Perú - INTE-PUCP, Perú

\section{RESUMEN}

En América Latina y el mundo, los pequeños agricultores, cazadores y pescadores tradicionales/artesanales contribuyen entre el 60\% a 70\% de la producción total, a la alimentación, y cocinas locales, regionales y mundial (Altieri \& Parviz, 2008; Oxfam, 2011). La mistura de sabores, saberes, cocinas locales y regionales anida en el archipiélago de la diversidad biocultural local y regional. Las cocinas locales y regionales pueden verse y posicionarse estratégicamente como un resurgir identitario íntimamente vinculado a la consciente regeneración y crianza del paisaje biocultural espiritual, y como parte de una propuesta alimentaria sostenible. Este artículo, desde una visión panorámica, reflexiona sobre eslabones claves de una cadena que surge como parte de un paradigma alternativo; además, contribuye a potenciar el estudio multidimensional de las cocinas en relación con las culturas, ecología y medio ambiente. Se concluye que, una estrategia sostenible turismococina-medioambiente-alimentación, es posible. Allí las pequeñas agriculturas son estratégicas por su potencial biocultural ecoespiritual. Contribuirían de manera realista a resolver problemas críticos como la desnutrición, la seguridad y calidad de la alimentación y regeneración del paisaje -local y regional- biocultural. Ello potenciaría, expandiría y agregaría valor al turismo sostenible en Perú.

Palabras clave: Ecoturismo, diversidad biocultural, sostenible, agriculturas.

\begin{abstract}
Worldwide and in Latin America small farmers, hunters, pastoralists, and fisherfolk communities contribute between 60 to 70 percent of the total food output (Oxfam, 2011; Altieri \& Parviz, 2008). Thus, serving the needs of local, regional and global kitchens. The mixture of taste, saberes, and local and regional kitchens nests in the local and regional bio-cultural diversity's archipelago. Local and regional kitchens can be observed
\end{abstract}

Este es un artículo Open Access bajo la licencia Creative Commons Atribución-NoComercial-Compartirlgual 4.0

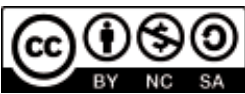


and positioned strategically as an emerging identity stream closely linked to the conscious regeneration of the bio-cultural-spiritual landscape, and as a part of a sustainable food proposal. From a panoramic view, this article reflects on key links of a chain that constitutes an alternative paradigm; and contributes to enhance local and regional kitchens multidimensionality with relation to culture, ecology and environment. It concludes that, a sustainable tourism-kitchen, environment-food strategy is possible due to the strategic centrality of small agricultures and their unique ancient bio-cultural-eco-spiritual potential. Small farming can contribute realistically to resolve critical problems of malnourishment, food safety and quality, and the regeneration of the local and regional bio-cultural landscape. That would potentiate, expand, and add value to sustainable tourism in Peru.

Keywords: Ecotourism, biocultural diversity, sustainable, agricultures.

\section{Introducción}

Frente a la actual crisis social, política, económica, espiritual y ambiental de la modernidad y el desarrollo urgen enfoques resilientes y sostenibles en los sectores claves de la política de Estado y de los negocios. La sostenibilidad es un tema central, local y global. Un enfoque, innovador, integrador, sostenible y multidimensional puede contribuir a potenciar el valor de las rutas locales y regionales turístico-gastronómicas/arte culinario ${ }^{1}$ agroecológico-ambiental-biocultural²-espirituales. Así como avanzar en la reconciliación campo-ciudad; ser humano-naturaleza-espiritualidad (Pilgrim \& Pretty, 2010). Es decir, en la refundación de una ética alimentario turística con profundo sabor plurinacional/étnico.

En las pasadas siete décadas la multidimensionalidad agroecológico-ambiental-cultural no ha sido articulada por el paradigma dominante unidimensional en las ciencias sociales, ni la política estatal y gobiernos locales y regionales.

Asistimos a la crisis global y fin de la era de la modernidad y el desarrollo capitalista como fue propuesta a fines de 1949 por el presidente de los Estados Unidos, Harry Truman. La crisis de la modernidad y el desarrollo ya era visible en 1970. En ese momento y después, estudios serios concluían que la modernidad y el desarrollo no es para todos (Esteva, Babones, \& Babcicky, 2013).

La crisis de la modernidad y el desarrollo es una manifestación de una situación más profunda: la crisis epistémica del pensamiento euroamericano centrado. Es decir, la crisis de un modo de ver, generar conocimiento entender y de actuar en el mundo (local, regional, nacional e internacional) (Gonzales, 2016; Mignolo, 2013; Wallerstein, 2006).

1 Como parte de la descolonización de la mesa y las cocinas, y dado que no existen abstracciones universales, usamos «gastronómicos», en plural, para reconocer la pluralidad epistemológica y ontológica del término. La expresión «arte culinario» se acerca más a las cocinas locales populares e Indígenas, en la medida que la preparación de los alimentos depende mucho de las culturas y conocimientos locales.

2 El trabajo académico sobre Diversidad Biocultural, DBC, de la Dra. Luisa Maffi's (2001) es conocido mundialmente. Este concepto enfoca la interdependencia de la Diversidad Biológica y Cultural. La DBC vincula lengua, conocimiento, y el medio ambiente. 


\section{Modernidad, universidad, crísis epistémica y antropoceno}

Esta crisis epistémica se expresa en el antropoceno. El antropoceno se asocia con el comienzo de la revolución industrial en Inglaterra en 1750. Este término reconoce un hecho sin precedentes, el impacto global de las sociedades industriales y capitalistas en la geología de la tierra y los ecosistemas. Entre los siglos XVIII y XX las universidades (kantiana y luego humboldtiana y más recientemente la neoliberal) continuaron apostando por la modernidad. Es decir, por la modernización del campo y la ciudad, lo urbano y lo rural, desde la unidimensionalidad (Anthropocene, 2017; Mignolo, 2013; Wallerstein, 2006).

\section{El desarrollo crea el subdesarrollo}

Sin duda, el presidente Truman inauguró en 1949, un momento singular en la historia de la modernidad en el siglo XX, al declarar que el desarrollo podía ser compartido y beneficiar al resto del mundo. Esta declaración automáticamente convirtió a dos mil millones de personas en subdesarrolladas. Universidad, capital, ciencia, y tecnología, y un concierto de organismos supranacionales (p. ej., Naciones Unidas), se convirtieron en los pilares de la profundización y expansión de la globalización de la modernidad y el desarrollo. Estos pilares contribuyeron a transformar el paisaje rural, población, agricultores, semillas y agriculturas (Esteva, Babones, \& Babcicky, 2013; Kloppenburg, 2004).

\section{La Universidad}

La universidad euroamericana, entre otros centros de generación y validación del conocimiento, ha jugado y juega un papel importante en la modernidad y el desarrollo. Durante el siglo XIX primero surgió la universidad kantiana que más tarde entró en tensión con la emergente universidad humboldtiana. Esta última se expandió por Europa y se convertió en el modelo internacional de universidad moderna. Durante la caída de los poderes imperiales europeos y la emergencia de Estados Unidos como el poder hegemónico mundial en 1949, y su actual crisis hegemónica, la universidad moderna eurocéntrica es reconocida como la autoridad indiscutida validadora del conocimiento. El pensamiento eurocéntrico-americano se autoirrogó la autoridad universal/mundial de validar lo que era/es y -no era/es- conocimiento; de lo que gustas al degustar; de lo que es y no es arte, de lo que es civilizado o no. Todo esto, hoy está en cuestión desde dentro y fuera del pensamiento euroamericano (postmodernidad, estudios culturales, estudios postcoloniales, estudios Indígenas, descolonialidad, y las otras epistemologías) (Avilés, 2017; De Sousa, 2012; Gonzales, 2015, 2016; Mignolo, 2013, 2014b; Wallerstein, 2006).

\section{Europeos y eurocentrismo}

Latinoamericanos y europeos tienen derecho a ser eurocéntricos. No se puede culpar al eurocentrismo de la ausencia en la universidad peruana de programas de sociología, en ciencias sociales y humanas en general, donde se enseñe y estudie sobre la comida, plantas medicinales y medicina tradicional; alimentación y genealogías del pensamiento latinoamericano. Desde la multidimensionalidad, pluriculturalidad y diversidad epistémica. Ni

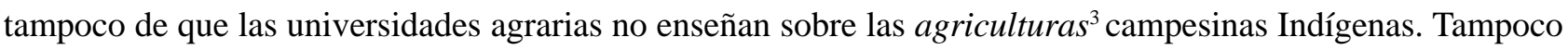

3 El término agriculturas se usa en este artículo para enfatizar la rica e intima asociación entre naturaleza y cultura. De manera específica para revalorar los complejos innovadores, y únicos sistemas de conocimiento y tecnologías campesinas locales (Sain \& Calvo, 2009; Gonzales \& Gonzalez, 2010). 
de porqué, los campesinos, que saben sobre estas agriculturas, no enseñan y colaboran como pares en tales universidades (Apffel-Marglin, 2002; Dabashi, 2015; Gonzales, 2015, 2016; Pariona, 2017; Wallerstein, 2016).

\section{Boom gastronómico en las Américas es parte de movimiento identitario y reconexión con la madre tierra}

El boom gastronómico en Perú es parte del resurgimiento en las Américas, de la Nueva Cocina Indoamericana.

En Norteamérica la incorporación de los otros saberes/conocimientos de las otras epistemologías o epistemologías del sur, se debió al movimiento por los derechos civiles (Civil Rights Movement). A ello, se debe que la universidad abrió sus puertas a los estudios asiáticos, americanos, afroamericanos, latinoamericanos, y nativo americanos. Esta apertura no significó una resolución fundamental a los múltiples problemas que dichas «minorías étnicas» continúan enfrentando dentro y fuera de la universidad. Hoy en Estados Unidos y Canadá, se viene dando un resurgimiento de las comidas nativas desde dentro y fuera de las comunidades nativo americanas. La Novo American Indian Cuisine, jóvenes chefs Indígenas, publicaciones y nuevos restaurantes en esta línea, son parte de un movimiento que crece día a día en alianza con otros movimientos, como por ejemplo, Slow Food America. Vale mencionar el caso de los chefs Lois Ellen Frank, Ph. D. (Kiowa) y Walter Whitewater (Diné/Navajo), en Estados Unidos. Así como el trabajo de Winona LaDuke, entre otros, Food as Medicine. Aparte de la producción de académicos nativo-americanos sobre el tema.

\section{Soñar, comer, degustar y pensar como un europeo o norteamericano}

El pensamiento moderno euroamericano centrado impartido en las universidades ha permeado muchas instituciones supranacionales y nacionales de manera casi natural. Para nuestras elites, para quienes trabajan en la mayoría de instituciones nacionales y supranacionales y sociedad en general, Europa y Estados Unidos eran, y aún, son el modelo por seguir (Wallerstein, 2006).

\section{Naciones Unidas y el paradigma euroamericano de desarrollo}

Hasta no hace mucho tiempo gran parte de la historia de los organismos de las Naciones Unidas promovían consecuentemente, en sus estudios, reportes y diagnósticos, la unidimensionalidad del conocimiento-paradigmas -modelos euroamericanos, para promover la modernidad. Cabe mencionar, los organismos de Naciones Unidas vinculados a la educación, ciencia y cultura (UNESCO); la agricultura (FAO), el medio ambiente (UNEP); la salud (WHO); y el desarrollo (UNDP). Esta tendencia dominante está cambiando lentamente en Naciones Unidas por una serie de factores que no corresponde explicar aquí por razones de espacio. Por ejemplo, en UNESCO, el programa denominado Local and Indigenous Knowledge Systems (LINKS); y en la FAO, Fondo Internacional para el Desarrollo Agrícola (FIDA), y el programa Sistemas Importantes del Patrimonio Agrícola Mundial (SIPAM) (FAO, 2017). Esto es solo el inicio de algo que ya se percibe en el horizonte (Tengo, 2012). Declarar a los territorios, tierras y culturas Indígenas y locales, patrimonio de la humanidad y comunidades líderes de la resiliencia, sostenibilidad y de la multidimensionalidad. 


\section{¿Estamos «todos» en piloto automático y en caída libre?}

El precio de entrar a la modernidad hegemónica ha exigido y exige usar y explotar de manera racional el espacio y los recursos naturales. También requiere la homogenización de la economía, agricultura, las semillas, la nación, la ciudadanía, la educación, la salud, el abandono de las tierras y territorios donde anidan y florecen la biodiversidad de la mano con los conocimientos, sabores y saberes de las agriculturas vivas de las poblaciones locales y pueblos originarios. Este uso racional de las economías extractivas muestra su devastador impacto a escala mundial en la transformación del paisaje local, remoción de poblaciones y despojo de sus territorios ancestrales; así como, contaminación profunda y global de los elementos fundamentales de la vida humana y del planeta.

La moral y la ética ecológico-ambiental parecen no tener mayor influencia, ni importancia en el desenfrenado ritmo extractivo denominado por algunos como ecocidio (Leuridan, 2016; Rumrrill, 2012). El Estado y los gobiernos latinoamericanos parecen seguir por inercia el modelo dominante de la modernidad neoliberal y las demandas insaciables del mundo global y de las transnacionales. Nos preguntamos, a partir de nuestra realidad pluricultural en Perú ¿tienen los actores e instituciones claves de la sociedad capacidad de pensar y proponer alternativas frente a ese fenómeno extractivista?

\section{El Sur epistémico, pluriversidad y multidimensionalidad}

Desde 1500 y desde el Sur epistémico se inició el largo, frustrante y penoso camino de continuar y afirmar la pluriversidad y la multidimensionalidad. Este persistente caminar marca un hito histórico el año 2007 con el reconocimiento y aprobación, luego de diez años de debates, de la Declaración de los Derechos de los Pueblos Indígenas de las Naciones Unidas. Es decir, el derecho a no pasar a la modernidad despojándose y/o siendo despojado de los elementos constitutivos de la identidad cultural de la tierra y el territorio. La declaración posibilita otras «modernidades» y «desarrollos» que se podrían denominar «Desarrollo étnico», «Desarrollo con cultura» o «Desarrollo con identidad y cultura» (Charters \& Stavenhagen, 2009; Esteva, Babones, \& Babcicky, 2013; Tauli-Corpuz, 2010; Stavenhagen, 1990).

\section{Universidad, desarrollo y turismo sostenible. Urgen nuevos lenguajes, conceptos, y maneras de mirar y relacionarse con el mundo}

En el contexto de la crisis global de la modernidad y el desarrollo. Frente a los retos de la propuesta más reciente de Naciones Unidas de un desarrollo sustentable y frente al «capitalismo salvaje» extractivista, ¿qué papel podría jugar la universidad con relación a otro(s) tipo(s) de desarrollo? (Tauli-Corpuz, 2010; Gonzales, 2015; Grillo, 1991; Mignolo, 2013, 2014a).

Durante el auge y crisis de la modernidad el binomio sociología-universidad (ciencias sociales y humanas incluidas) apostó por la unidimensionalidad y la visión monocultural euroamericana centrada. Las teorías y metodologías del Norte epistémico (liberales, neoliberales, modernistas, postmodernistas, marxistas) han predominado y aún predominan en la comprensión, incidencia política, recomendaciones de políticas y políticas, sobre los temas claves de la sociedad (p. ej., agricultura, suficiencia alimentaria, sistemas alimentarios, ecología, 
cultura, salud, economía, mercado, saberes e identidad). Es decir, ¿con qué lenguaje(s) y concepto(s) vemos, entendemos y actuamos sobre nuestra realidad? ¿Cómo superamos la percepción, entendimiento y transformación de nuestra realidad en la perspectiva de una(s) economía(s) sustentable(s), más allá del eufemismo del desarrollo neoliberal sustentable? (De Sousa, 2014; Grillo, 1991; Mignolo, 2013, 2014a, 2016; Wallerstein, 2006).

La búsqueda y necesidad urgente de un(os) lenguaje(s), concepto(s) y visión(es) del mundo nos obliga, a todos los actores centrales involucrados, a mirar en todas las direcciones, dentro y fuera del país pluricultural, con una perspectiva de diálogo intercultural e interdisciplinario. Desde la universidad, desde el Estado y gobiernos locales y regionales, y desde organismos supranacionales de diverso nivel (Parlamento Andino, Naciones Unidas, Banco Mundial, Banco Interamericano, Cooperación Internacional, etc) (Mignolo, 2014; Wallerstein, 2006).

\section{Otros desarrollos y paradigmas son posibles}

En lo que concierne al tema de este artículo la búsqueda de otros paradigmas, lenguajes, conceptos y visiones nos remiten a volver nuestra mirada hacia lo local y hacia adentro (Alatas, 2004; Grillo, 1991, 1990; Mignolo, 2013). Es decir, hacia nuestro ser y conciencia interior, y hacia las poblaciones locales que viven en un contacto más íntimo con su entorno -la tierra, la naturaleza y el cosmos- (Avilés, 2017; Toledo \& Barrera-Bassols, 2008). Ese volver nuestra mirada no es una práctica regular, ni masiva entre nosotros. La modernidad y el desarrollo nos ha obligado y obliga a estar más en la vorágine del día a día del mundo de afuera. Constatamos que la modernidad y el desarrollo no han beneficiado a todos los peruanos medianamente. El mapa de pobreza persiste. El desarrollo y modernidad urbana y rural muestra serias disparidades sociales y económicas: salud, educación, calidad del aire, agua, transporte, calidad de vida, calidad del entorno y medio ambiente. El presidente Kuczinksy nos recordó el pasado 28 de Julio que tan solo el 16\% de las aguas servidas (relaves mineros, desagües industriales y domésticos) son tratadas en plantas de tratamiento. El otro $84 \%$ contaminado va directamente a los ríos, lagos, lagunas y al mar.

\section{¿Cuál es la riqueza de lo local?}

Una serie de estudios y proyectos evidencian -a pesar de ciertos sesgos etnocéntricos- que aún hay riqueza cultural, ecoambiental y espiritual, tangible e intangible, en las poblaciones locales y tradicionales, Indígenas, originarias y campesinas. En sus cocinas, saberes y sabores locales y regionales. Para potenciar lo local parte de la respuesta está en enfocar sus potencialidades y en su (re)posicionamiento ecológico - ambiental - cultural estratégico. Es necesario trascender la visión del paradigma neoliberal dominante de la modernización y el desarrollo sobre lo local y sus poblaciones. El trascender promueve que nuestra mirada e intención sea respetuosa e incluyente de las localidades/comunidades y sujetos donde anidan las cosmovisiones, los otros conoceres, sentires, saberes y sabores que dan y han dado forma al paisaje biocultural-espiritual (Toledo \& Barrera-Bassols, 2008). 


\section{Modernidad, agriculturas marginales y patrimonio cultural}

Los excluidos o marginados de la modernidad son en muchos casos la otra cara de lo que se va reconociendo de manera segmentada como parte del patrimonio cultural, patrimonio tangible e intangible. Las poblaciones locales, originarias, Indígenas, campesinas conforman el vivo y dinámico tejido biocultural y Sur epistémico, el cual se funda, nutre y emerge de la íntima relación con diversos paisajes locales bioculturales. Este conglomerado epistémico-cultural se está regenerando con y sin ayuda externa (estatal, cooperación internacional). Este archipiélago es el que puede constituirse en los eslabones que contribuyan vigorizados al desarrollo con identidad y cultura. Es decir, a la regeneración y reconexión del paisaje bioecológico-gastronómicocultural-espiritual sostenible (Gonzales, 2015, 2016; Pimbert 1994).

En resumen, estaríamos frente a los elementos constitutivos de un paradigma alternativo al propuesto hasta la fecha desde arriba, por una mayoría de actores, nacionales y extranjeros. Esta mayoría puede contribuir a este paradigma alternativo, redireccionando el enfoque metodológico y apoyo financiero. Así la oferta turístico gastronómica local y regional incorporaría tres elementos descuidados por la modernidad y el desarrollo naturaleza, cultura y ecología. Esta triada es parte de una estrategia integrada de manejo sostenible-territorialmultidimensional para superar el mapa de pobreza local, regional y nacional.

\section{Posicionando las cocinas, sabores y saberes locales y regionales del Sur epistémico}

Norte y Sur epistémico, no son regiones geográficas. Son opciones de vida, agriculturas, prácticas, paradigmas y sistemas de conocimiento que se encuentran en el norte, sur, este y oeste geográficos. He aquí el reto y oportunidad para la universidad del siglo XXI, transitar de la universidad a la multiversidad. De la universalidad a la pluriversidad de la epistemología y ontología monocultural excluyente y singular, a la inclusión, respeto y trato de pares con las otras epistemologías y ontologías, en plural. Incluyendo la forja de proyectos de ley y ejecución de políticas que fortalezcan la investigación y dialogo de conocimientos, saberes y sabores locales, regionales y nacionales. Ello facilitaría una relación más estrecha, pragmática y mutuamente beneficiosa entre la universidad y lo local/comunal en el siglo XXI. Queda así planteado el reto del reconocimiento de lo multidimensional y multifuncional, multidisciplinario, transdisciplinario y no disciplinario como parte de reconstituir y forjar nuestras propias genealogías de conocimientos que sustenten las culturas educativas localescomunitarias, sus sabores y saberes (De Sousa, 2014).

Es en el contexto arriba presentado donde ubicamos y posicionamos las cocinas, sabores y saberes, locales y regionales del Sur epistémico. En particular del Perú. Al cambiar los términos del diálogo, es posible soñar, recordar, recuperar, regenerar, potenciar, y (re)crear las rutas ecológico-cultural-gastronómico-espirituales sostenibles. Así como las agriculturas, tecnologías; calendarios ritual-agrofestivos, la estética y aestésis de las prácticas y cultivos agropastoriles, y otros ingredientes de la diversa y rica culinaria en cuestión. Ello sería posible al sensibilizarnos y en cómo nos acercarnos respetuosamente para aprender de nuestras genealogías de pensamiento y saberes local y regional vivos (Gonzales, 2000; Sain \& Calvo, 2009). 


\section{Es posible lo imposible. Otras maneras de vivir y relacionarnos son posibles}

Lo imposible siempre fue posible con voluntad política inclusiva de cambio de paradigma/conceptual. El paradigma y la dimensión global de la crisis nos reta urgentemente a ser creativos, respetuosos e incluyentes. Las actuales desarmonías requieren un trabajo individual y colectivo. Los desequilibrios sociales, ecológicos, medio ambientales involucran a todos, conscientemente, a reeducarnos, a aprender a desaprender, a respetarnos para poder respetar, a reconciliarse con sí mismo para poder reconciliarnos, amarse a sí mismo para poder amar, a reconectarnos interiormente para poder reconectarnos entre todos y con la vida toda. En la visión de los pueblos originarios andinos lo anterior se resume en los siguientes principios que anidan en la cosmovisión andina: reciprocidad, sacralidad de la vida, respeto mutuo, criar y dejarse criar, y procurar constante balance y armonía con todo y entre todos - tangible e intangible- (Avilés, 2017; Grillo, 1990; Rengifo, 1991a, 1991b).

\section{Cambio climático y pequeña agricultura}

El cambio climático se registra en una serie de estudios climático-geológico-oceanográficos. Este cambio dramático está íntimamente asociado con el Antropoceno, políticas de desarrollo y el crecimiento económico. Los escenarios de incremento de un grado de la temperatura global son críticos con relación a la agricultura en la región andina. La Evaluación Global y Sub Global para América Latina del International Assessment of Agricultural Knowledge, Science and Technology for Development (IAASTD, 2009) reconoce este fenómeno y su impacto en la agricultura, entre otros (United Nations Environmental Programme - UNEP, 2009; Programa de Adaptación al Cambio Climático - PACC Perú, 2009; Comunidad Andina, 2008).

Estudios de Altieri y Koohafkan (2008), Oxfam (2011) y FAO (2017) para Latinoamérica reconocen la importancia y contribución de la pequeña agricultura y la cosmovisión andina hoy y en el futuro para la agricultura en nuestra región. Así como la necesidad urgente de que los tres sistemas de producción agrícola (convencional, agroecológico y campesino Indígena) en la región transiten hacia la sostenibilidad. Esto, se entiende dado el exceso de énfasis y optimismo que los gobiernos dieron al fenómeno conocido como la «revolución verde». Es decir, la aplicación de la ciencia, la tecnología y el uso indiscriminado de insumos derivados del petróleo, desde 1950 en la producción agrícola. Hoy, sabemos que este paradigma agrícola está en crisis pues el petróleo no es un recurso infinito. Aparte de las consecuencias de su explotación y uso para el efecto invernadero, el medioambiente, la naturaleza, poblaciones Indígenas y población en general.

\section{Menús alternativos sostenibles y sanos: agroecológico y campesino Indígena}

Frente a la agricultura convencional y los serios problemas que ocasiona a la vida, todas las agriculturas ecológicas y campesinas Indígenas son alternativas reales y viables (IAASTD, 2009). Estas ofrecen mayores posibilidades para un medioambiente donde el agua, suelos y tierra, microorganismos, el aire, la vida campesina, la naturaleza y los cultivos sean saludables y contribuyan a la salud de todos y entre todos. Estas agriculturas emergentes se vienen fortaleciendo con y sin apoyo externo, desde hace más de veinte años, a partir de varias experiencias comunitarias de base (Chirapaq, Proyecto Andino de Tecnologías Campesinas - PRATEC, Asociación Andes, CEPROSI-Cusco, Asociacion Bartolomé Aripaylla-Ayacucho). 
Sin duda, una evaluación y sistematización de las experiencias citadas y otras, pueden contribuir a incrementar la escala e impacto a nivel intercomunal, distrital, provincial y regional dentro de un paradigma de desarrollo con identidad y cultura. Este (re)posicionamiento y fortalecimiento permitiría un diálogo intercultural, de aprendizaje mutuo, respetuoso y entre equivalentes, con actores externos (p. ej., cooperación internacional, gobiernos regional y local, ONG, ministerios, FAO y Naciones Unidas, Banco Mundial, BID, IICA, entre otros.)

\section{Armando el rompecabezas de los estudios sobre comida, alimentación y cocinas sustentables en el Perú}

Una rápida revisión de la bibliografía sobre este tema, durante los últimos veinte años, nos muestra:

1. en general un número significativo de libros y artículos, y

2. los enfoques son de distinta latitud y profundidad.

Con cargo a una revisión más exhaustiva cabe resaltar el trabajo, por más de dos décadas, del Grupo de cocinas locales y regionales de la Universidad San Martín de Porres, bajo el liderazgo activo y productivo de la investigadora, empresaria y chef, Isabel Álvarez.

Álvarez y el Grupo de trabajo de cocinas locales y regionales de la USMP han levantado a pulso un sólido andamiaje multidimensional que contribuye a restablecer e incorporar en el discurso y la investigación sociológica, así como en las políticas ministeriales, las múltiples conexiones de la comida, cocinas y sus vínculos íntimos con la identidad, cosmovisiones, sabores, saberes, ritos, espiritualidad, calendarios gastronómicos, calendarios rituales agrofestivos, cosmovisiones (Álvarez, 2013; Valladolid, 2017).

No examinamos aquí las múltiples implicaciones del trabajo de Alvarez y el grupo que lidera. Sí reconocemos que dicho trabajo se inscribe en el marco global de la crisis de civilización y cambios de paradigmas en la cultura de la alimentación. Reconocer este marco global facilita avanzar en la investigación y reconexiones del tema en cuestión desde la multidimensionalidad. Entre otros temas a reconocer y reconectar están: agriculturas sostenibles-epistemologías-sistemas alimentarios-ordenamiento y manejo territorial-resiliencia-ética -ecología- ociedad civil-Estado-políticas-cocinas y comida. En otras palabras, cómo esta cadena nos lleva al ámbito de una nueva sociología de la alimentación y la comida que estudia y evalúa a genealogías de pensamiento y paradigmas, en crisis y alternativos, locales, regionales y nacionales. Nos lleva también a la política de lo ecoespiritual de la alimentación, la comida y la cocina. Es decir, a restablecer y regenerar un balance de la complejidad biocultural-ecológica-espiritual desarticulada y velada por la modernidad en crisis (Maffi, 2001).

\section{Regeneración y crianza de la mega biodiversidad, del paisaje biocultural espiritual desde la cosmovisión andina vigente}

La región Andino Amazónica es mundialmente reconocida como un hotspot de megabiodiversidad. Los Andes peruanos constituyen un centro de origen de agrobiodiversidad. La vida en los Andes aún es agrocéntrica. Reconociendo los sesgos etnocéntricos de los estudios de geografía, arqueología, antropología y etnohistoria 
andina, estos todavía contribuyen a reconocer la sabia y milenaria adaptación del ser humano andino al majestuoso paisaje de los Andes (Posey, 1999).

Desde una vertiente no académica disciplinaria reconocemos importantes contribuciones sobre el mundo andino tanto por intelectuales desprofesionalizados, como por miembros pertenecientes a asociaciones de comunidades campesinas andinas. Estas genealogías de pensamiento muestran en diverso grado las particularidades de la relación comunidad humana andina con su entorno natural y cósmico (Gonzales, 2014, 2015; Mignolo, 2013, 2014).

A la luz de las prioridades del paradigma neoliberal de desarrollo agropecuario estatal y su limitada relación con las Comunidades Campesinas (CC), es preciso no idealizar a las 6277 que existen en el Perú. Sin embargo, en los últimos veinte años se han dado importantes experiencias locales de pequeña escala de CC de desarrollo con identidad y cultura. Estas han recibido fondos, en pequeña escala, de agencias de la cooperación internacional. Estas experiencias demuestran que ha llegado el momento de aplicarlas a una escala mayor y con financiamientos de mayor envergadura.

La potencial contribución de las CC a una estrategia alimentaria, y a rutas turístico ecológica ambiental resilientes y sostenibles de las CC se multiplicaría desde otros paradigmas alternativos al estatal neoliberal, al grueso de la cooperación internacional, y organismos internacionales de desarrollo. Esto tendría múltiples efectos beneficiosos. Por ejemplo, beneficiaría a las cocinas locales y regionales y añadiría un valor ecoambientalcultural-espiritual a los circuitos gastronómicos, que complementarían otros circuitos y paradigmas turísticos para diversos tipos de turistas.

\section{Una matriz ecoambiental-cultural-espiritual alternativa}

La narrativa de los párrafos anteriores da una respuesta a dos preguntas. ¿Cuál es el problema? ¿Cuál es la causa? A continuación, respondemos la pregunta ¿cuál es la solución?

El sabio físico Albert Einstein expresó una vez: «Cada uno es un genio. Pero si juzgas a un pez por su habilidad de trepar un árbol, vivirá toda su vida creyendo que es un estúpido». En otra ocasión declaró: «El esquema mental que ha creado el problema no es parte de la solución».

En virtud de los objetivos del milenio de la ONU y siete décadas de modelos poco exitosos de modernización y desarrollo del sector rural en el Perú recogemos, en una síntesis apretada, un modelo informado y consolidado por varias experiencias locales con y por pueblos originarios campesinos, ubicados a lo largo y ancho de los Andes Peruanos y en Bolivia. Algunas de estas experiencias de más de veinte años requieren ser sistematizadas y sintetizadas. En su conjunto, estas ofrecen refinados enfoques, metodologías y técnicas culturalmente sensibles y consensuadas por los participantes vía sus mecanismos tradicionales (Delgado \& Delgado, 2014; Gonzales, 2011, 2015, 2016)

Regenerar el paisaje biocultural (Maffi, 2001) y sus cocinas locales y regionales en Perú requiere de una matriz que sustente visiones sustentables del mundo local con apertura a la colaboración externa y uso adaptado 
de tecnologías externas. Esta matriz ha sido probada a lo largo de varios años de diferentes maneras por PRATEC, Chirapaq y Agruco, se adapta a las ocho regiones naturales y a las 103 zonas ecológicas existentes en el país.

Elaborar esta matriz ha sido posible al reconocer que es necesario:

1. Fortalecer la crianza del pacha local (chacras/campos de cultivo, montañas y pastos naturales) vía el trabajo comunal (ayni, minga, choba choba).

2. Fortalecer la crianza orgánica del ayllu por medio de la gobernanza tradicional (autoridades tradicionales).

3. Recuperar el respeto y amor por las deidades y la madre naturaleza, vía el fortalecimiento de la crianza de rituales y festividades.

4. Fortalecer los espacios regionales (los caminos de las semillas), donde la diversidad de las semillas y los saberes se regeneran por medio de visitas entre campesinos a nivel local, regional y nacional.

La matriz conceptual se funda en la cosmovisión andina. Esta cosmovisión a su vez informa el cómo actuar y relacionarse en y con el mundo, y con el paisaje biocultural espiritual. En el vivir cotidiano a lo largo del ciclo agropastoril y del calendario ritual agrofestivo se pone en práctica la cosmovisión. Esta práctica a su vez retroalimenta la cosmovisión. Es decir, la cosmovisión andina se reafirma, persiste y trasciende por la práctica de las poblaciones andinas que, a pesar de la migración y la violencia, histórica y contemporánea, aún son eminentemente agrocéntricas. El potencial de la aplicación de la matriz conceptual se aprecia en la Tabla 2 para el caso de la región Sallqa/Jallca o Puna, una de las ocho ecoregiones andinas.

Como se señaló, la flexibilidad y adaptabilidad de la matriz, junto a otros métodos y técnicas participativas, permite trabajar con las ocho regiones, sus respectivos ecosistemas y temas claves (conservación de la biodiversidad; manejo del territorio y ecosistemas, seguridad alimentaria y estrategias de adaptación al cambio climático). Cualesquiera de los puntos de entrada, educación, conservación de la biodiversidad, implican un proceso de afimación cultural local y regional, cuyo último objetivo es la regeneración del paisaje biológico cultural en su conjunto. Es decir, la vida en su multidimensionalidad. La vida toda. Tangible e intangible (Gonzales, 2011; Gonzales, 2014, 2015; IAASTD, 2019; Ishizawa, Rengifo, \& Arnillas, 2010).

\section{Conclusiones}

Una estrategia sostenible turismo-cocina-medioambiente-alimentación, es posible. Allí las pequeñas agriculturas sostenibles y resilientes son estratégicas por su potencial productivo biocultural-ecológico-espiritual. La matriz conceptual fundamentada en la cosmovisión andina contribuye y contribuiría aún más, de manera realista, a resolver problemas críticos como la desnutrición, la seguridad y calidad de la alimentación, de la vida y el medio ambiente; al procurar la regeneración y armonía del paisaje, local y regional, biocultural. Ello potenciaría, expandiría y agregaría valor al turismo sostenible local y regional en Perú. 
Tabla 1

Matriz general. Estrategias comunitarias de adaptación al cambio climático

\begin{tabular}{cccc}
\hline & \multicolumn{3}{c}{ Escenarios } \\
\cline { 2 - 4 } Componentes & $\begin{array}{c}\text { Paisaje biocultural } \\
\text { de la Cuenca }\end{array}$ & $\begin{array}{c}\text { Chacra (Actividad } \\
\text { productiva) }\end{array}$ & $\begin{array}{c}\text { Casa y } \\
\text { comida }\end{array}$ \\
\hline
\end{tabular}

1. Infraestructura

2. Organizativo

3. Rituales y festividades

4. Diálogo con la naturaleza

Fuente y elaboración Rengifo, 2010

Tabla 2

Estrategias adaptativas tradicionales para el cambio climático en el paisaje biocultural andino de la Jallca o Puna

\begin{tabular}{|c|c|c|c|c|}
\hline Componentes $\begin{array}{c}\text { Eaisaje } \\
\text { biocultural }\end{array}$ & $\begin{array}{c}\text { Crianza del } \\
\text { agua }\end{array}$ & $\begin{array}{c}\text { Crianza de los } \\
\text { montes }\end{array}$ & $\begin{array}{l}\text { Crianza de los } \\
\text { cerros tutelares }\end{array}$ & $\begin{array}{c}\text { Crianza de la } \\
\text { pradera }\end{array}$ \\
\hline Infraestructura & Cosecha de agua. & Clausura de montes. & $\begin{array}{l}\text { Construcción de } \\
\text { cruces y altares. }\end{array}$ & $\begin{array}{l}\text { Construcción de } \\
\text { sistemas de riego de } \\
\text { bojedales. } \\
\text { Viviendas temporales } \\
\text { en la Puna }\end{array}$ \\
\hline $\begin{array}{l}\text { Organizativos: familiares, } \\
\text { comunales e intercomunales }\end{array}$ & $\begin{array}{l}\text { Autoridad tradicional del } \\
\text { agua. }\end{array}$ & $\begin{array}{l}\text { Autoridades de los } \\
\text { montes. } \\
\text { Chaku de montes. }\end{array}$ & $\begin{array}{l}\text { Fortalecimiento de los } \\
\text { sacerdotes andinos. }\end{array}$ & $\begin{array}{l}\text { Autoridades de las } \\
\text { áreas de pastoreo } \\
\text { rotativo (Astanas). }\end{array}$ \\
\hline Rituales y fiestas & $\begin{array}{l}\text { Visitas ritual y fastiva a } \\
\text { los ojos de agua. } \\
\text { Consumo ritual del } \\
\text { agua de los puquios. }\end{array}$ & $\begin{array}{l}\text { Fiestas a los montes: } \\
\text { el vigawantuy. } \\
\text { Chaku ritual de árboles } \\
\text { y arbustos. }\end{array}$ & $\begin{array}{l}\text { Rituales a los apus y } \\
\text { achachilas. }\end{array}$ & $\begin{array}{l}\text { Rituales de permiso a } \\
\text { los apus. }\end{array}$ \\
\hline Diálogo con la naturaleza & & $\begin{array}{l}\text { Conversación con } \\
\text { floración de árboles y } \\
\text { arbustos }\end{array}$ & & $\begin{array}{l}\text { Conversación con } \\
\text { indicadores climáticos } \\
\text { de la pradera. }\end{array}$ \\
\hline
\end{tabular}

Fuente y elaboración Rengifo, 2010 


\section{Referencias}

Alatas, S. H. (2004). The Captive Mind and Creative Development. In P. N. Mukherji, \& Ch. Sengupta (Eds.), Indigeneity and Universality in Social Science. A South Asian Response (pp. 83-98). India, New Delhi: Sage Publications.

Altieri, M., \& Koohafkan, P. (2008). Enduring Farms: Climate Change, Smallholders and Traditional Farming Communities. Environment \& Development Series 6. Malaysia, Penang: Third World Network.

Álvarez, I. (2013). Reconociendo y revalorando las cocinas Regionales del Perú: Lima, sus otros sabores, Huacho, Huaral, Huarochirí y Canta (Vol. 3). Lima: Fondo Editorial Universidad de San Martin de Porres.

Anthropocene (2017). Welcome to the Anthropocene. Recuperado de https://www.youtube.com/watch?v=20dJDZphIhk

Apffel-Marglin, F. (2002). From Fieldwork to Mutual Learning: Working with PRATEC. Environmental Values, 11, $345-367$.

Avilés, M. (2017). No soy tu cholo. Lima: Debate.

Comunidad Andina (2008). El cambio climático no tiene fronteras. Impacto del cambio climático en la comunidad andina. Lima: Secretaria General de la Comunidad Andina.

Charters, C., \& Stavenhagen, R. (Eds.). (2009). Making the Declaration Work: The United Nations Declaration on the Rights of Indigenous Peoples. Copenhagen: IWGIA.

Dabashi, H. (2015). Can Non-Europeans Think? Chicago: University of Chicago Press and London, Zed Books.

Delgado, F., \& Delgado, M. (2014). Vivir y Comer Bien en los Andes Bolivianos. Aportes de los sistemas agroalimentarios y las estrategias de vida de las naciones Indígena originario campesinas a las políticas de seguridad y soberanía alimentaria. La Paz: Plural Editores.

De Sousa, B. (2012). The University at a Crossroads. Human Architecture: Journal of the Sociology of Self-Knowledge, 10(1), 7-16.

De Sousa, B. (2014). Epistemologies of the South: Justice against Epistemicide. Routledge.

Esteva, G., Babones, S., \& Babcicky, P. (2013). The Future of Development. A Radical Manifesto. Bristol: Policy Press.

FAO (2017). Sistemas Importantes del Patrimonio Agrícola Mundial. Recuperado de http://www.fao.org/giahs/es/

Gonzales, T. (2000). The Cultures of the Seed in the Peruvian Andes. En S. B. Brush (Ed), Genes in the Field: On-Farm Conservation of Crop Diversity. IDRC/IPGRI/Lewis Publishers.

Gonzales, T. (2011). Methodologies and Protocols followed by Andean Organizations: Andean Project for Peasant Technologies, (PRATEC), Agroecology University Cochabamba, (AGRUCO), and Andes Association (ANDES). Report to the Indigenous Partnership for Agrobiodiversity and Food Sovereignty, (IPAF), Rome, Italy.

Gonzales, T. (2014). Kawsay (Buen Vivir) y afirmación cultural: Pratec-Naca, un paradigma alternativo en los Andes. En B. Marañón (Coord.), Buen Vivir y descolonialidad. Crítica al desarrollo y la racionalidad instrumentales (pp. 121-139). México: Universidad Nacional Autónoma de México.

Gonzales, T. (2015). An Andean Indigenous Autonomous Community-Based Model for Knowledge Production. Special issue on Indigenous Peoples Experiences of Autonomy in Latin America. Latin American and Caribbean Ethnic Studies Journal, 10(1), 107-133.

Gonzales, T., \& Gonzalez, M. (2010). From colonial encounter to decolonizing encounters. Culture and Nature seen from the Andean Cosmovision of Ever: The Nurturance of Life as Whole. In J. Pretty \& S. Pilgrim (Eds.), Nature and Culture (pp. 83-101). London: Earthscan publishers.

Grillo, E. (1990). Cultura y Agricultura Andina. En Agruco \& Pratec (Eds.), Agroecología y Saber Andino. Lima: PRATEC.

Grillo, E. (1991). El Lenguaje en las Culturas Andina y Occidental Moderna. En Cultura Andina Agrocéntrica. Proyecto Andino de Tecnologías Campesinas. Lima: PRATEC.

International Assessment of Agricultural Knowledge, Science and Technology for Development - IAASTD (2009). Agriculture at a Crossroads: Volume III. International Assessment of Agricultural Science and Technology for Development Latin America and the Caribbean. Washington, DC: Island Press. 
Ishizawa, J., Rengifo, G., \& Arnillas, N. (2010). Gestión de Proyectos Incrementales. La Experiencia Fondo de Iniciativas de Afirmación Cultural, (FIAC)2002-2009. Lima: PRATEC.

Maffi, L. (2001). On Biocultural Diversity: Linking language, knowledge, and the environment. Washington, DC: Smithsonian Institution Press.

Mignolo, W. (2013). Historias locales/diseños globales. Colonialidad, conocimientos subalternos y pensamiento fronterizo. Madrid: Ediciones Akal.

Mignolo, W. (2014a). Educación y decolonialidad: aprender a desaprender para poder re-aprender. Un diálogo geopolítico-pedagógico con Walter Mignolo, entrevista por Facundo Giuliano y Daniel Berisso. Revista del IICE, 35, 61-71.

Mignolo, W. (2014b). Spirit out of bounds returns to the East: The closing of the social sciences and the opening of independent thoughts. Current Sociology, 62, 584.

Mignolo, W. (2016). Sustainable Development or Sustainable Economies? Ideas Towards Living in Harmony and Plenitude. India: Dialogue of Civilizations Research Institute.

Oxfam (2011). Who will Feed the World? The production challenge. Oxfam research report. Oxfam.

Pariona, W. (2017). Hampiq. Salud y Emfermedad en Ayacucho. Universidad Nacional de San Cristobal de Huamanga: Pakarina Ediciones.

Pilgrim, S., \& Pretty, J. (2010). Nature and Culture. U.K.: Earthscan Publishers.

Pimbert, M. (1994). Editorial. Etnoecológica, 2(3), 3-5.

Posey, D. A. (1999). Introduction: Culture and Nature-The Inextricable Link. En D. Posey (ed.), Cultural and Spiritual Values of Biodiversity. UNEP's Global Biodiversity Assessment Volume. Cambridge: University Press.

Programa de Adaptación al Cambio Climático - PACC Perú (2009). Adapting to Climate Change in the Andes. The Climate Change Adaptation Programme. Perú: PACC.

Rengifo, G. (1991a). El Saber en la cultura andina y en occidente moderno. En PRATEC, Cultura Andina Agrocéntrica. Proyecto Andino de Tecnologías Campesinas, (pp. 99-130). Lima: PRATEC.

Rengifo, G. (1991b). Prueba y diálogo en la cultura andina. Experimentación y extensión en occidente moderno. En PRATEC, Cultura Andina Agrocéntrica. Proyecto Andino de Tecnologías Campesinas (pp. 209-242). Lima: PRATEC.

Rengifo, G. (2010). Programa Regional Titikaka. Cambio Climático, suficiencia alimentaria y saber local. Apuntes para un programa de acompañamiento. Lima: PRATEC.

Rumrill, R. (2012). Defender y Promover a la Pequeña Producción Agraria frente a la Neolatifundización del Planeta. Selva de Ideas. Agronoticias, 377. Recuperado de http://www.agronoticiasperu.com/377/selvadeideas-377.htm

Sain, G., \& Calvo, G. (2009). Agri-Culturas de América Latina y el Caribe. Elementos para una contribución de la ciencia y la tecnología al desarrollo sustentable. San José, CR: IICA, UNESCO.

Stavenhagen, R. (1990). The Ethnic Question. United Nations: University Press.

Tauli-Corpuz, V. (2010). Indigenous Peoples’ Self-Determined Development: Challenges and Trajectories. In V. Tauli-Corpuz, L. EnkiweAbayao, \& R. de Chavez (Eds.), Towards an Alternative Development Paradigm. Indigenous Peoples Selfdetermined Development, (pp. 1-78). El Baguio: Valley Printing Specialist.

Tengö, M. (10-13 de abril de 2012). (compiladora). Conocimiento para el Siglo 21. Conocimiento Indígena, Conocimiento Tradicional, y Ciencia. Conectando Diversos Sistemas de Conocimiento. Taller de Diálogo. Guna Yala: Panamá.

Toledo, V., \& Barrera-Bassols, N. (2008). La memoria biocultural. La importancia de las sabidurías tradicionales. España: Icaria Editorial.

United Nations Environmental Programme - UNEP (2009). Latin America and the Caribbean. Environment Outlook, GEO LAC 3. United Nations: Environmental Programme.

Wallerstein, I. (2006). Abrir las Ciencias Sociales. En I. Wallerstein (Coord.), Informe de la Comisión Gulbenkian para la reestructuración de las ciencias sociales. Mexico: Siglo XXI. 


\section{Bibliografía recomendada}

Agronoticias (2012). Habla el Ministro de Agricultura y Riego, Eco. Milton von Hesse. Comunidades Campesinas y Nativas según el IV Cenagro. Recuperado de http://www.agronoticiasperu.com/393/perspectivas393-2.htm

Gonzales, T. (1987). Political Economy of Agricultural Research and Education in Peru, 1902- 1909. M.S.: University of Wisconsin.

Gonzales, T. (1996). Political ecology of peasantry, the seed, and NGOs in Latin America: A study of Mexico and Peru, $1940-1996$. (Ph.D. thesis. University of Wisconsin, Madison).

Gonzales, T., Chambi, N., \& Machaca, M. (1999). Agricultures and cosmovision in contemporary Andes. En Cultural and Spiritual Values of Biodiversity. Londres: Intermediate Technology Publications.

Mato, D. (2015). Pueblos Indígenas, Estados y educación superior. Cuadernos de Antropologia Social, 41, 5-23.

Pimbert, M. (1994a). The Need for Another Research Paradigm. Seedling, 11, 20-32. 